\title{
The importance of accurate anatomic assessment for the volumetric analysis of the amygdala
}

L. Bonilha,

E. Kobayashi,

F. Cendes

and L.M. Li
Laboratório de Neuroimagem, Departamento de Neurologia, Faculdade de Medicina, UNICAMP, Campinas, SP, Brasil

\section{Correspondence}

L.M. Li

Departamento de Neurologia Faculdade de Medicina, UNICAMP 13083-970 Campinas, SP Brasil

Fax: +55-19-3788-7483

E-mail: limin@fcm.unicamp.br

Research supported by FAPESP (No. 00/04710-2)

Received August 3, 2003 Accepted January 6, 2005 Accepted january 6, 2005

\section{Abstract}

There is a wide range of values reported in volumetric studies of the amygdala. The use of single plane thick magnetic resonance imaging (MRI) may prevent the correct visualization of anatomic landmarks and yield imprecise results. To assess whether there is a difference between volumetric analysis of the amygdala performed with single plane MRI 3-mm slices and with multiplanar analysis of MRI 1-mm slices, we studied healthy subjects and patients with

Key words - Amygdala

- Magnetic resonance

- Volumetry

- Epilepsy

- Temporal lobe temporal lobe epilepsy. We performed manual delineation of the amygdala on T1-weighted inversion recovery, 3-mm coronal slices and manual delineation of the amygdala on three-dimensional volumetric T1-weighted images with $1-\mathrm{mm}$ slice thickness. The data were compared using a dependent $t$-test. There was a significant difference between the volumes obtained by the coronal planebased measurements and the volumes obtained by three-dimensional analysis $(\mathrm{P}<0.001)$. An incorrect estimate of the amygdala volume may preclude a correct analysis of the biological effects of alterations in amygdala volume. Three-dimensional analysis is preferred because it is based on more extensive anatomical assessment and the results are similar to those obtained in post-mortem studies.

\section{Introduction}

The amygdala is responsible for defining the intensity of emotional responses, for determining targets to which responses are directed and for promoting behavioral and visceral alterations in response to emotionally burdening stimuli. It also plays an important role in autonomic responses, emotional behavior, food intake, arousal, and sexual and motor activity (1). Neuroimaging studies have demonstrated the role of the amygdala in emotional recognition concerning memory
$(2,3)$, fear conditioning (4), facial expression $(5,6)$, and auditory stimuli $(2,7)$. This is believed to be a consequence of its function of formation of long-term memory associated with emotionally arousing events $(2,3)$.

In vivo assessment of the amygdala volume by magnetic resonance imaging (MRI) has demonstrated some alterations in certain subsets of clinical populations. Reduction of the volume of the amygdala has been shown to be able to lateralize patients with temporal lobe epilepsy $(8,9)$, and has also been shown to occur in recurrent depression (10), obses- 
sive-compulsive disorder (11), Alzheimer disease (12), and in male subjects with schizophrenia (13). Conversely, patients with autism (14), bipolar-affective disorder and female patients with schizophrenia show an increased amygdala volume (13). Some of these results, however, have not been confirmed and are still far from definitive.

A striking feature of studies that have addressed the measurement of the amygdala is the wide range of volumes encountered, with reports of volumes ranging from 1 to almost $4 \mathrm{~cm}^{3}$ in normal subjects $(15,16)$. Another striking finding is the number of discrepancies concerning the landmarks adopted for manual segmentation alongside with different MRI scanning protocols. Presumably the volumetric assessment of such a small structure may be impaired by the use of a low-resolution image based on a thick section or by an unfavorable section orientation. Moreover, the gray matter composing the amygdaloid complex usually merges with the gray matter of the striatum, further challenging accurate segmentation. Therefore, it is believed that many volumetric studies of the amygdala have overestimated its volume (17), since it is known from post-mortem studies that the average amygdala volume ranges from 1.1 to $1.6 \mathrm{~cm}^{3}$ (7).

To test the hypothesis of whether there is any significant difference between volumetric studies performed with different imaging quality and different accuracy of anatomic visualization, we compared the data obtained with two different methods using the same landmarks for the amygdala boundaries.

\section{Material and Methods}

\section{Subject assessment}

We studied 15 healthy subjects (13 women). All subjects had an unremarkable previous medical history, without any neurological or psychiatric events.

We also studied 15 patients ( 9 women) with drug-refractory temporal lobe epilepsy (TLE) without any other co-morbid condition. The patients with TLE had unilateral hippocampal atrophy, with seizure onset being recorded on the side of atrophy.

The Ethics Committee of our institution approved the study and all subjects signed an informed written consent to participate in the study.

\section{Magnetic resonance imaging}

The subjects underwent a standardized MRI protocol (18) consisting of coronal 3$\mathrm{mm} \mathrm{T1}$ and volumetric T1 with isotropic voxel acquisition.

The coronal images were acquired perpendicular to the long axis of the hippocampus, defined by the sagittal images (T1weighted inversion recovery (IR), 3-mm thick, flip angle $=200^{\circ}, \mathrm{TR}=2,800 \mathrm{~ms}$, TE $=14 \mathrm{~ms}$, inversion time $=840 \mathrm{~ms}$, matrix $=$ $130 \times 256, \mathrm{FOV}=16 \times 18 \mathrm{~cm})$. The sequence acquisition time was approximately $10 \mathrm{~min}$.

The volumetric T1-weighted images with 1 -mm isotropic voxel were obtained using a three-dimensional (3-D) spoiled gradient-echo acquisition with sagittal volume excitation $\left(\mathrm{TR}=18, \mathrm{TE}=10\right.$, flip angle $=30^{\circ}, \mathrm{FOV}=$ 256 x 204, 1-mm sagittal slices). This sequence acquisition time was approximately $14 \mathrm{~min}$. The images were submitted to postacquisition processing; the raw images were first converted to MINC format and then corrected for field non-homogeneity using N3 (19). Finally, the images underwent linear stereotaxic transformation into a standard space (20).

\section{Volumetric analysis}

The anatomic landmarks used for segmentation of 3-mm coronal images and volumetric 1-mm images were identical (Table 1). The segmentation steps for each method are outlined below.

Coronal plane-based segmentation. We 
used the NIH Image software package which allows image visualization and magnification, with the possibility of manual delineation of regions of interest. The quantitative analysis was performed on T1-weighted IR, 3-mm coronal planes.

A single observer (E. Kobayashi) determined the volumes in this protocol. We referred to previous MRI-based studies for anatomic boundaries (21) (Figure 1A).

The anterior limit of the amygdala was defined in the anteriormost slices containing the temporal lobe stem. The inferior limit was set at the white matter of the angular bundle in more rostral sections. In more caudal sections, the postero-inferior limit was defined by the presence of the hippocampus, which appears as a gray matter mass inferior and lateral to the amygdala. The alveus or the uncal recess of the lateral ventricle was used for separation of the amygdala from the hippocampal head. The lateral limit was considered to be the transition of the gray matter and the white matter of the temporal lobe stem; the mesial limit was defined on the pial surface, superior to the gyrus semiannularis and the angular bundle, inferior to the gyrus semiannularis. The superior limit was defined as an arbitrary line from the quadrigeminal cistern to the inferior limit of the circular sulcus of the insula. The volumes obtained were further corrected for the total intracranial volume (22).

$3-D$ segmentation. We used the DISPLAY software package developed at the Brain Imaging Centre of the Montreal Neurological Institute, which creates electronically linked reformatted images on the coronal and axial plane, displays the three planes together, and also permits regions of interest enlargement. Delineating anatomical boundaries is also facilitated by contrast adjustment and the possibility for navigation through an isotropic voxel of $1 \mathrm{~mm}$ in different orientations with the same resolution. The software automatically calculates the volumes of labeled structures. In this protocol the vol-
Table 1. Anatomical landmarks adopted for amygdala segmentation.

Limits of the amygdala

Anterior Starts at the level of the lateral sulcus closure

Lateral Lateral portion (the most lateral pixels) of the semi-circle drawn on the axial plane

Medial Located one row of pixels lateral to the pial surface located inferiorly from the semiannularis sulcus. In the sections posterior to the hippocampal fissure, it is located on the pial surface, and at the posterior end, in the mesial protuberance of the hippocampus head

Superior Defined by a line between the endorhinal sulcus and the fundus of the inferior portion of the circular sulcus of the insula

Inferior Defined by a line between the fundus of the semiannularis sulcus (or the mid-point of the pial surface between the uncal cleft and the endorhinal sulcus) and the inferior horn of the lateral ventricle, or defined by the intersection of the pia with the line that forms a continuum with the white matter of the angular bundle

Posterior Ends at the point where gray matter first started to appear superior to the alveus and lateral to the hippocampal head
A

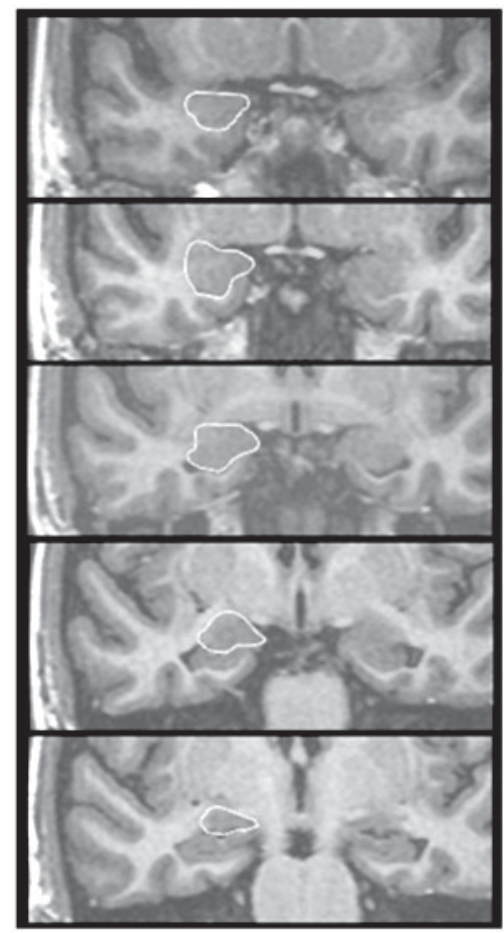

Figure 1. A, The amygdala delineated in 3-mm T1-inversion recovery magnetic resonance imaging (MRI) in its rostrocaudal extension. $B$, The amygdala delineated in 1-mm T1 MRI on the coronal, axial and sagittal planes, respectively.

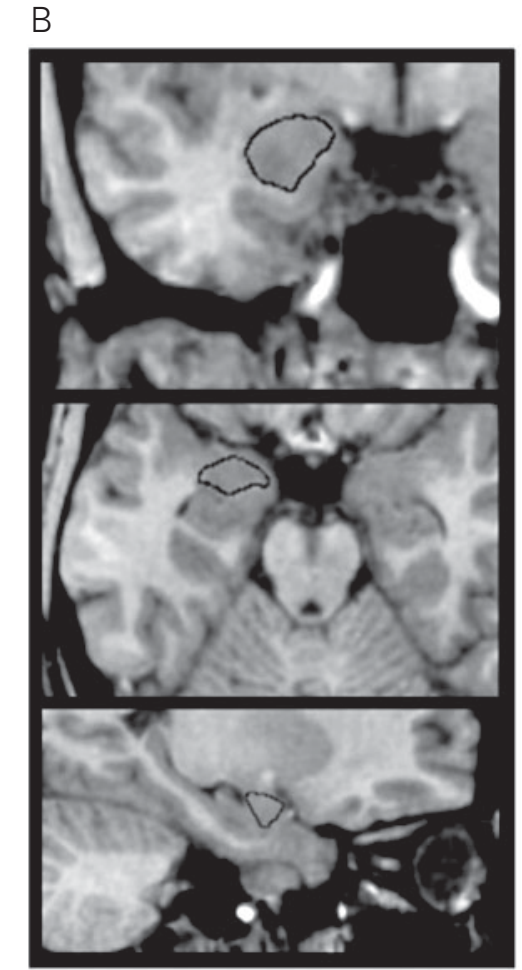

B 
Figure 2. Box and whisker plots of the volumes of the right and left amygdala of control subjects $(A)$ and patients with TLE (B), for each method of segmentation. $3 \mathrm{~mm}=$ coronal plane-based analysis; $1 \mathrm{~mm}=$ multiplanar analysis; L-TLE, RTLE = left and right temporal lobe epilepsy, respectively. umes were determined by a single observer (L. Bonilha). We used as reference a previously described 3-D-based method for hippocampus and amygdala segmentation (15), as well as reviewed temporal lobe functional and structural studies (23) (Figure 1B).

Different from the coronal plane segmentation, the horizontal plane was the mainstay for the segmentation of the amygdala in 3-D images. The sagittal and coronal planes were used for constant reference, but we felt that the best way to define the postero-inferior limits of the amygdala was to visualize the alveus and the lateral ventricle on the horizontal plane by image magnification. Whenever there was difficulty with the definition of the alveus, we referred to the region of the head of the hippocampus displayed on the sagittal plane. One row of pixels was added to the hippocampus in the alveus region. After the definition of the postero-inferior limits, the anterior limits were defined by a semi-circle drawn anteriorly with its radius defined at the midpoint of the line drawn over the alveus in the posterior border. Special care was taken not to involve the entorhinal cortex by avoiding the inclusion of the most anterior and medial row of pixels, or by segmenting the entorhinal cortex in advance. Finally, after segmentation on the horizontal plane, a thorough rostrocaudal analysis was made, with particular attention paid to the superior limit, defined as a horizontal line passing by the most lateral portion of the

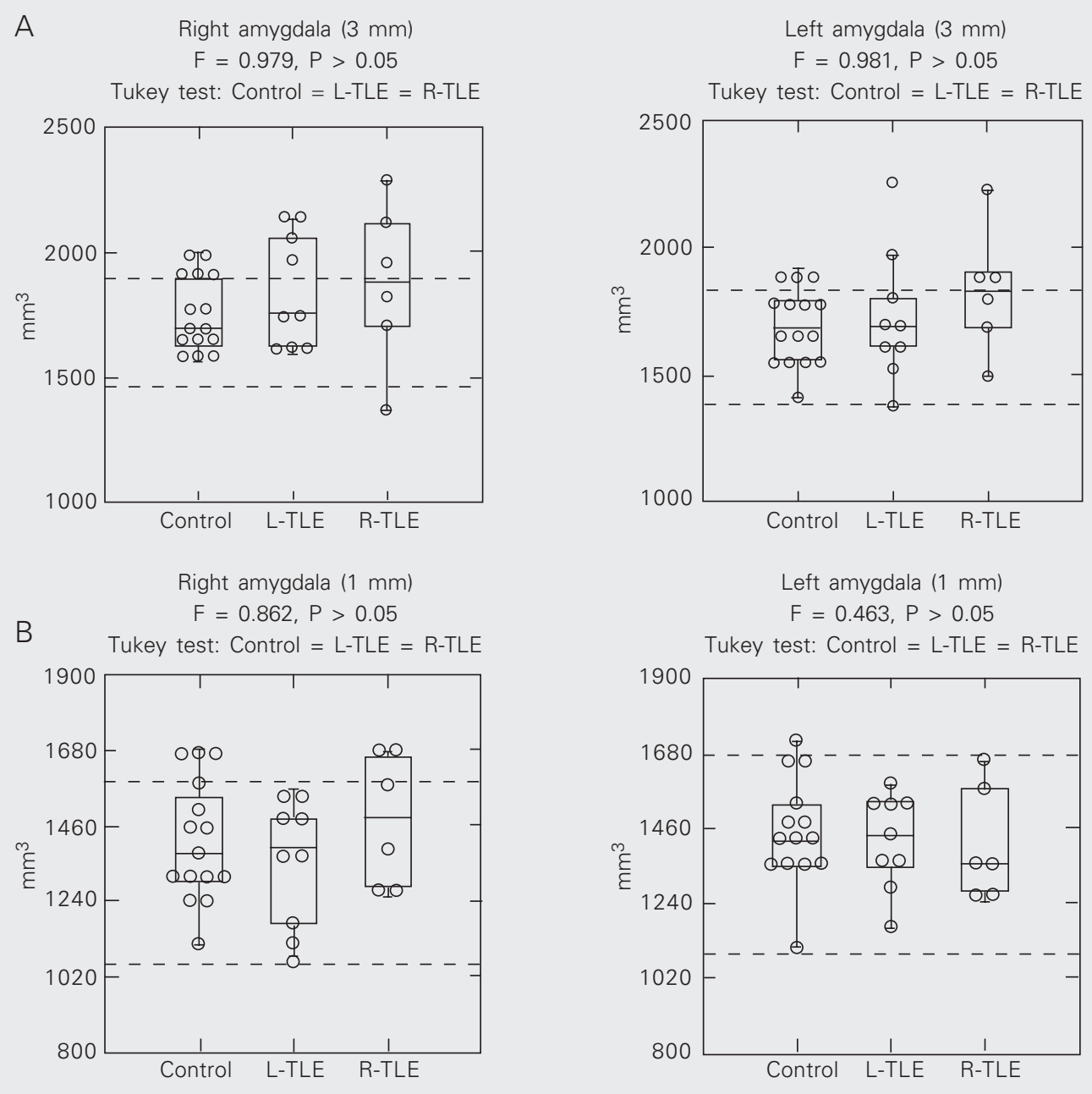


quadrigeminal cistern. The rostral end of the hippocampus started to appear infero-lateral to the amygdala in more caudal sections, and served as a landmark.

\section{Reliability assessment}

Intraobserver reliability was evaluated by random selection of 5 subjects and by repeating the segmentation of all structures twice. Inter-rater reliability was assessed by comparison of the volumes of the amygdala of five randomly chosen subjects obtained by 3-D segmentation by the two observers (L. Bonilha and E. Kobayashi) who were not aware of each other's results. Reliability was assessed using the repeatability coefficient adopted by The British Standards Institution (24).

\section{Statistical analysis}

The volumes of the structures analyzed are reported as means $\pm \mathrm{SD}$ for TLE patients and for the control group. The group of TLE patients was subdivided according to the side of hippocampal atrophy. Data were assessed for normal distribution by the KolmogorovSmirnov test. For comparison of the volumes obtained by the two different forms of analysis we used a dependent $t$-test. We also performed analysis of variance (ANOVA) with Tukey post hoc comparison for the evaluation of putative differences between the volume of the right and left amygdala among controls and patients with right and left TLE, for each form of measurement. The level of significance was set at $\mathrm{P}<0.05$ in all analyses.

\section{Results}

We evaluated 15 patients with chronic drug-refractory TLE (6 men) and 15 normal subjects $(2$ men). All patients with TLE presented unilateral hippocampal atrophy and lateralization of seizures based on ictal re- cording that was concordant with the side of the hippocampal atrophy. Nine patients had left TLE and 6 had right TLE. The volumes obtained by coronal plane segmentation and by 3-D segmentation followed normal distribution according to the Kolmogorov-Smirnov test $(\mathrm{P}>0.05)$.

\section{Coronal plane-based segmentation}

The mean volume and standard deviation (SD) of the right amygdala of the control subjects was $1742 \pm 151 \mathrm{~mm}^{3}$, and the mean volume and SD of the left amygdala was $1703 \pm 146 \mathrm{~mm}^{3}$. Among the TLE patients, the mean volumes and SD obtained for patients with right TLE were: right amygdala $=$ $1875 \pm 323 \mathrm{~mm}^{3}$ and left amygdala $=1829 \pm$ $245 \mathrm{~mm}^{3}$, and for patients with left TLE the values were: right amygdala $=1842 \pm 225$ $\mathrm{mm}^{3}$ and left amygdala $=1733 \pm 258 \mathrm{~mm}^{3}$. Dependent $t$-tests applied to the values of the coronal-based segmentation did not indicate asymmetry between controls $(\mathrm{t}(14)=1.316$, $\mathrm{P}>0.05)$, patients with left TLE $(\mathrm{t}(8)=$ $1.244, \mathrm{P}>0.05)$ and patients with right TLE $(\mathrm{t}(5)=0.768, \mathrm{P}>0.05)$. ANOVA did not show a significant difference in amygdala volume between controls and patients with TLE (Figure 2A), with $>95 \%$ power. The asymmetry index $(=[100 \cdot(\mathrm{R}-\mathrm{L})] /[(\mathrm{R}+\mathrm{L}) /$ 2]) also did not differ between patients and controls, with $>95 \%$ power.

\section{Three-dimensional segmentation}

For the controls, the mean volume and SD was $1459 \pm 229 \mathrm{~mm}^{3}$ for the left amygdala and $1413 \pm 199 \mathrm{~mm}^{3}$ for the right amygdala. Among the TLE patients, the mean volumes and SD obtained for right TLE subjects were $1410 \pm 167 \mathrm{~mm}^{3}$ for the left amygdala and $1473 \pm 189 \mathrm{~mm}^{3}$ for right amygdala, and the values obtained for left TLE patients were $1422 \pm 141 \mathrm{~mm}^{3}$ for the left amygdala and $1349 \pm 188 \mathrm{~mm}^{3}$ for the right amygdala. Dependent $t$-tests applied to 
the values of 3-D segmentation did not show asymmetry between the controls $(\mathrm{t}(14)=$ $1.731, \mathrm{P}>0.05)$, patients with left TLE ( $\mathrm{t}(8)$ $=0.839, \mathrm{P}>0.05)$ and patients with right TLE $(\mathrm{t}(5)=-0.584, \mathrm{P}>0.05)$. ANOVA did not show a significant difference in the volume of the amygdala between controls and patients with TLE, with $>95 \%$ power (Figure 2B). The asymmetry index also did not differ between patients and controls, with $>95 \%$ power.

\section{Reliability assessment}

There was no significant difference between the two measurements performed by one rater or by two raters, since the differences between the two measurements were less than 2 SD (intraobserver assessment, left amygdala repeatability coefficient $=366$; right amygdala repeatability coefficient $=$ 538 ; intraobserver assessment, left amygdala repeatability coefficient $=486$; right amygdala repeatability coefficient $=240$ ) .

\section{Comparison between coronal plane-based segmentation and 3-D analysis (Figure 3)}

The dependent $t$-test showed difference between the volumes obtained by the coronal plane-based measurements and the volumes obtained by the 3-D analysis of the right and left amygdala among controls (right amygdala: $\mathrm{t}(14)=6.832, \mathrm{P}<0.001$; left amygdala: $\mathrm{t}(14)=4.814, \mathrm{P}<0.001)$; of the right and left amygdala among patients with right TLE (right amygdala: $\mathrm{t}(5)=2.668, \mathrm{P}<0.05$; left amygdala: $\mathrm{t}(5)=3.328, \mathrm{P}<0.05)$, and of the right and left amygdala among patients with left TLE (right amygdala: $\mathrm{t}(8)=5.756, \mathrm{P}<$ 0.001 ; left amygdala: $\mathrm{t}(8)=2.956, \mathrm{P}<0.05$ ).

\section{Discussion}

We compared the results obtained by manual segmentation of the amygdala performed with 3-D analysis of 1-mm T1 weighted MRI slices and performed with analysis of coronal 3-mm T1-IR-weighted MRI slices and observed a significant difference in the volumes obtained from these two methods of analysis.

The volumetric analysis of the amygdala is a difficult task. It is difficult to accurately separate the amygdaloid nuclei from the surrounding gray matter, particularly to exclude the entorhinal cortex, the perirhinal cortex and the hippocampal head from the region of interest (15).

Most of the MRI volumetric studies of the amygdala were performed on coronal plane images. In order to overcome the difficulties of anatomical visualization posed by the indistinct borders of the amygdala, a number of different segmentation protocols have been developed to increase the accuracy of delineation and to exclude the hippocampal formation, the entorhinal cortex, the tail of the caudate nucleus, and the claustrum in the region ascribed to the amygdala.

The methods developed for the definition of the anatomic boundaries of the amygdala on the coronal plane can be enumerated and summarized as follows (17): first - performing the manual delineation of the amygdala in a pre-set number of slices in order to exclude the hippocampal head; second - defining the boundaries of the amygdala based on mesial temporal lobe landmarks or extratemporal landmarks; third - joint analysis of digital reformatted planes other than the coronal plane for constant reference of anatomic landmarks.

The quantitative MRI studies of the amygdala performed to date have shown different values. It is believed that the studies performed using coronal plane MRI have overestimated the actual size of the amygdala. Post-mortem studies have reported that the amygdala volume ranges from 1.1 to $1.6 \mathrm{~cm}^{3}$ after correction for fixation-related shrinkage, but different (25-27) volumetric MRI analyses have reported values ranging from 1 to near $4 \mathrm{~cm}^{3}(15,17-28)$. 

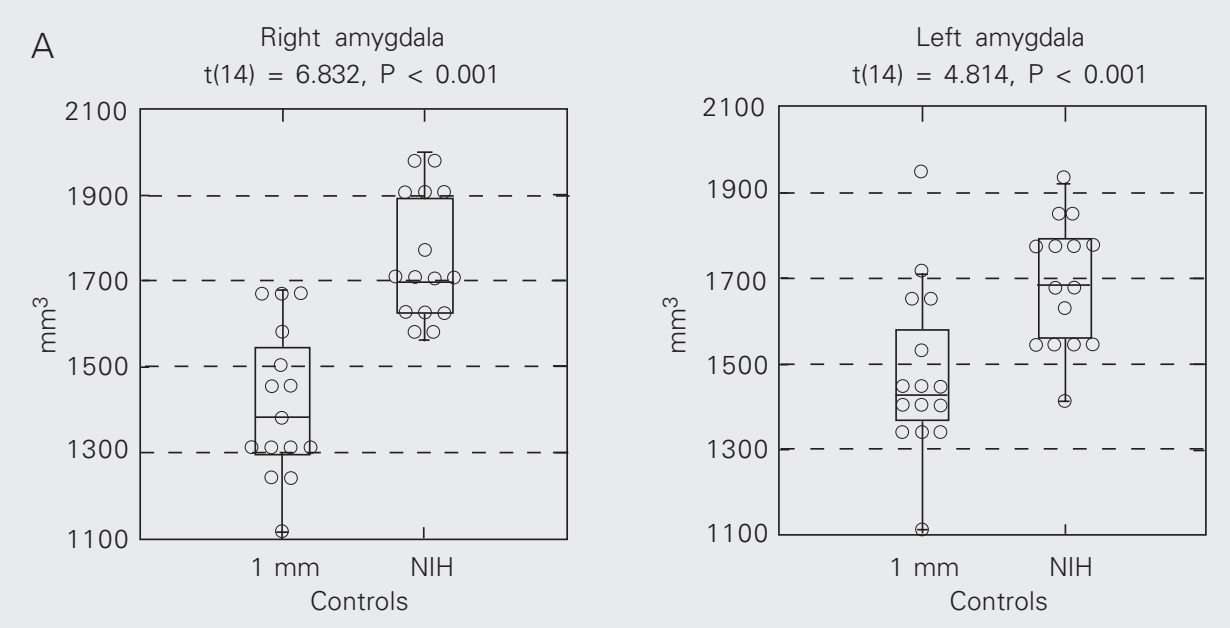

Figure 3. Box and whisker plots of amygdala volumes of controls (A), right TLE (B) and left TLE (C) measure on coronal plane-based segmentation and multiplanar analysis. Legend: 3 $\mathrm{mm}$ - coronal plane based analysis, $1 \mathrm{~mm}$ - multiplanar analysis. TLE $=$ temporal lobe epilepsy; $\mathrm{NIH}=\mathrm{NIH}$ image, National Institutes of Health software package.

B

Right amygdala $t(5)=2.668, P<0.05$
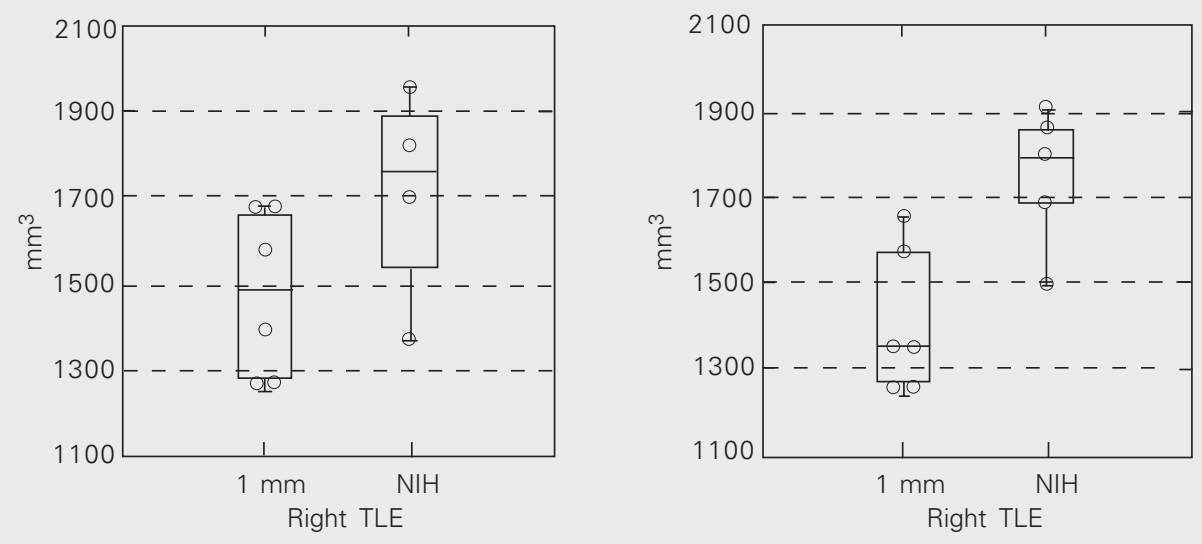

C

Right amygdala $t(8)=5.756, P<0.001$
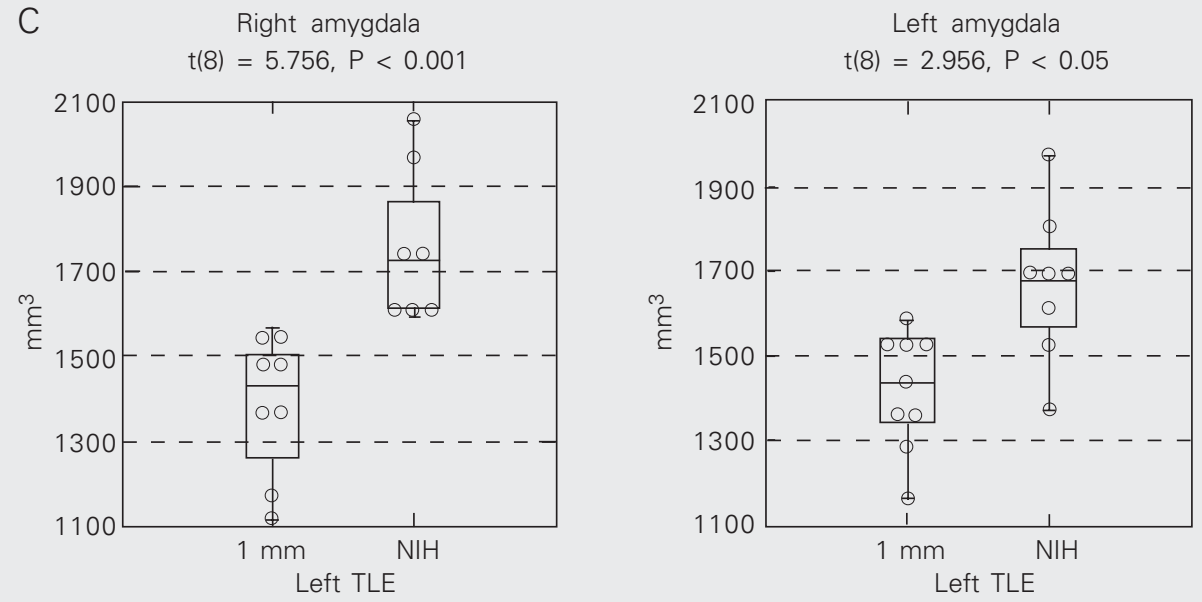
The accuracy of the volumetric MRI study of the amygdala is influenced by some factors. First: the possibility of unequivocal definition of anatomic landmarks; second: the partial volume effect generated by thick MRI slices; third: the image post-processing linear transformation to the stereotaxic space.

It is possible to follow a strict anatomic protocol for analysis of the volume of the amygdala on coronal slices, but the concurrent 3-D analysis of the sagittal, coronal and axial planes facilitates the definition of anatomic boundaries and allows the visualization of discrete landmarks. Therefore, 3-D studies performed on reformatted images are considered to be more accurate, especially those with emphasis on the axial plane, where the separation of the hippocampus and the amygdala is performed by the visualization of the alveus and the lateral ventricle (15). Analysis of the axial plane also prevents underestimation of the anterior and mesial portion of the amygdala because it allows the discrimination of the perirhinal and entorhinal cortex. Therefore, the possibility of accurate visualization of the anatomical landmarks is possibly the key technical variable influencing the results obtained in the morphometric analysis of the amygdala.

The volumes observed in the 3-D study are closer to those observed in post-mortem histological studies, which have been proposed as a benchmark for evaluating the validity of MRI-derived volume estimates (17). The smaller volumes obtained with the 3-D method may reflect a more accurate definition of the transition between the hippocampus and the amygdala, and the exclusion of the region considered to belong to the entorhinal cortex. Conversely, the superior limit set as an arbitrary line from the quadrigeminal cistern to the inferior limit of the circular sulcus of the insula in order to prevent the inclusion of the basal nuclei in the region of interest can underestimate the amygdaloid volume by an incomplete inclusion of the corticomedial nucleus. However, the definition of the superior border was the same for the two forms of measurement and was not responsible for the differences obtained. On the basis of the similar results obtained by histological analysis and 3-D evaluation, the straightforward definition of the superior limit is an advantageous strategy and the eventual exclusion of the corticomedial nucleus may be inexpressive.

The 3-D study is performed with images linearly transformed to the stereotaxic space, and this may be an additional factor accounting for the difference between the results, since the coronal-based evaluation uses images that were not spatially normalized and its volumes are corrected by the mean head size. However, the spatial normalization and volume correction by the intracranial volume have been used interchangeably in the literature $(22,29,30)$ and a difference between them has not been formally assessed.

The partial volume effect is another technical variable that could explain the differences observed between the forms of measurement, since each one is performed on MRI of different thickness. It has been shown that the partial volume effect significantly influences the morphometric analysis of small brain structures such as the entorhinal cortex (31), but does not influence the morphometric analysis of larger brain structures such as the hippocampus (32). Accordingly, the partial volume effect of the morphometric analysis of the amygdala is not particularly influential (33).

Since the volumes obtained by $3-\mathrm{D}$ analysis are different from the values obtained by thick MR coronal plane MRI, this may have influenced the conclusions of previous clinical studies carried out to investigate the presence of amygdaloid volume alterations, and may have caused misinterpretation of amygdaloid changes by inaccurate analysis of its boundaries.

Among the different technical aspects that can explain the differences in morphometric analysis of the amygdala, the anatomi- 
cal definition is the most important variable (33). Hence, the differences observed in this study are due to the different possibilities of accurately defining anatomical landmarks. 3-D analysis is based on a thorough anatomical analysis of the mesial temporal lobe region with more accurate definition of ana- tomical landmarks due to the constant reference to other MRI planes. Therefore, the use of reformatted planes of thin MRI with strict adherence to anatomical landmarks should be preferred for accurate quantitative analysis of the amygdala.

\section{References}

1. Afif A \& Bergman RA (1997). Functional Neuroanatomy: Text and Atlas. 1st edn. McGraw-Hill Professional Publishing, New York.

2. Cahill L, Haier RJ, Fallon J, Alkire MT, Tang C, Keator D, Wu J \& McGaugh JL (1996). Amygdala activity at encoding correlated with long-term, free recall of emotional information. Proceedings of the National Academy of Sciences, USA, 93: 8016-8021.

3. Cahill L, Babinsky R, Markowitsch HJ \& McGaugh JL (1995). The amygdala and emotional memory. Nature, 377: 295-296.

4. LaBar KS, LeDoux JE, Spencer DD \& Phelps EA (1995). Impaired fear conditioning following unilateral temporal lobectomy in humans. Journal of Neuroscience, 15: 6846-6855.

5. Adolphs R, Tranel D, Damasio H \& Damasio A (1994). Impaired recognition of emotion in facial expressions following bilateral damage to the human amygdala. Nature, 372: 669-672.

6. Young AW, Aggleton JP, Hellawell DJ, Johnson M, Broks P \& Hanley JR (1995). Face processing impairments after amygdalotomy. Brain, 118 (Part 1): 15-24.

7. Scott SK, Young AW, Calder AJ, Hellawell DJ, Aggleton JP \& Johnson M (1997). Impaired auditory recognition of fear and anger following bilateral amygdala lesions. Nature, 385: 254-257.

8. Guerreiro C, Cendes F, Li LM, Jones-Gotman M, Andermann F, Dubeau F, Piazzini A \& Feindel W (1999). Clinical patterns of patients with temporal lobe epilepsy and pure amygdalar atrophy. Epilepsia, 40: 453-461.

9. Kalviainen R, Salmenpera T, Partanen K, Vainio P, Riekkinen PSr \& Pitkanen A (1997). MRI volumetry and T2 relaxometry of the amygdala in newly diagnosed and chronic temporal lobe epilepsy. Epilepsy Research, 28: 39-50.

10. Sheline YI, Gado MH \& Price JL (1998). Amygdala core nuclei volumes are decreased in recurrent major depression. NeuroReport, 9: 2023-2028.

11. Szeszko PR, Robinson D, Alvir JM, Bilder RM, Lencz T, Ashtari M, Wu H \& Bogerts B (1999). Orbital frontal and amygdala volume reductions in obsessive-compulsive disorder. Archives of General Psychiatry, 56: 913-919.

12. Cuenod CA, Denys A, Michot JL, Jehenson P, Forette F, Kaplan D, Syrota A \& Boller F (1993). Amygdala atrophy in Alzheimer's disease. An in vivo magnetic resonance imaging study. Archives of Neurology, 50: 941-945.

13. Gur RE, Turetsky BI, Cowell PE, Finkelman C, Maany $\vee$, Grossman RI, Arnold SE, Bilker WB \& Gur RC (2000). Temporolimbic volume reductions in schizophrenia. Archives of General Psychiatry, 57: 769-775.

14. Howard MA, Cowell PE, Boucher J, Broks P, Mayes A, Farrant A \& Roberts N (2000). Convergent neuroanatomical and behavioural evidence of an amygdala hypothesis of autism. NeuroReport, 11: 2931-2935
15. Pruessner JC, Li LM, Serles W, Pruessner M, Collins DL, Kabani N Lupien S \& Evans AC (2000). Volumetry of hippocampus and amygdala with high-resolution MRI and three-dimensional analysis software: minimizing the discrepancies between laboratories. Cerebral Cortex, 10: 433-442.

16. Achten E, Boon P, De Poorter J, Calliauw L, Van De KT, De Reuck $J$ \& Kunnen M (1995). An MR protocol for presurgical evaluation of patients with complex partial seizures of temporal lobe origin. American Journal of Neuroradiology, 16: 1201-1213.

17. Convit A, McHugh P, Wolf OT, de Leon MJ, Bobinski M, De Sant S, Roche A \& Tsui W (1999). MRI volume of the amygdala: a reliable method allowing separation from the hippocampal formation. Psychiatry Research, 90: 113-123.

18. Kobayashi E, Lopes-Cendes I, Guerreiro CA, Sousa SC, Guerreiro MM \& Cendes F (2001). Seizure outcome and hippocampal atrophy in familial mesial temporal lobe epilepsy. Neurology, 56: 166172.

19. Sled JG, Zijdenbos AP \& Evans AC (1998). A nonparametric method for automatic correction of intensity nonuniformity in MRI data. IEEE Transactions on Medical Imaging, 17: 87-97.

20. Collins DL, Neelin P, Peters TM \& Evans AC (1994). Automatic 3D intersubject registration of MR volumetric data in standardized Talairach space. Journal of Computer Assisted Tomography, 18: 192-205.

21. Watson C, Andermann F, Gloor P, Jones-Gotman M, Peters $T$, Evans A, Olivier A, Melanson D \& Leroux G (1992). Anatomic basis of amygdaloid and hippocampal volume measurement by magnetic resonance imaging. Neurology, 42: 1743-1750.

22. Kobayashi E, Cendes F, Guerreiro CAM, Guerreiro MM \& LopesCendes I (1999). MRI abnormalities in familial temporal lobe epilepsy. Neurology, 52: A545 (Abstract).

23. Gloor P (1997). The Temporal Lobe and Limbic System. Oxford University Press, New York.

24. Bland JM \& Altman DG (1986). Statistical methods for assessing agreement between two methods of clinical measurement. Lancet, 1: 307-310.

25. Scott SA, DeKosky ST \& Scheff SW (1991). Volumetric atrophy of the amygdala in Alzheimer's disease: quantitative serial reconstruction. Neurology, 41: 351-356.

26. Bogerts B, Meertz E \& Schonfeldt-Bausch R (1985). Basal ganglia and limbic system pathology in schizophrenia. A morphometric study of brain volume and shrinkage. Archives of General Psychiatry, 42: 784-791.

27. Herzog AG \& Kemper TL (1980). Amygdaloid changes in aging and dementia. Archives of Neurology, 37: 625-629.

28. Achten E, Deblaere K, De Wagter C, Van Damme F, Boon P, De Reuck J \& Kunnen M (1998). Intra- and interobserver variability of 
MRI-based volume measurements of the hippocampus and amygdala using the manual ray-tracing method. Neuroradiology, 40: 558-566.

29. Bernasconi N, Bernasconi A, Caramanos Z, Antel SB, Andermann F \& Arnold DL (2003). Mesial temporal damage in temporal lobe epilepsy: a volumetric MRI study of the hippocampus, amygdala and parahippocampal region. Brain, 126: 462-469.

30. Bonilha L, Kobayashi E, Rorden C, Cendes F \& Li LM (2003). Medial temporal lobe atrophy in patients with refractory temporal lobe epilepsy. Journal of Neurology, Neurosurgery and Psychiatry,
74: 1627-1630.

31. Bonilha L, Kobayashi E, Cendes F \& Li LM (2003). Effects of method and MRI slice thickness on entorhinal cortex volumetry. NeuroReport, 14: 1291-1295.

32. Laakso MP, Juottonen K, Partanen K, Vainio P \& Soininen H (1997). $\mathrm{MRI}$ volumetry of the hippocampus: the effect of slice thickness on volume formation. Magnetic Resonance Imaging, 15: 263-265.

33. David AS, Brierley B \& Shaw P (2002). Measuring amygdala volume. British Journal of Psychiatry, 181: 255-256. 